\title{
КОМПЬЮТЕРНАЯ ОЧИСТКА АУДИОМАТЕРИАЛА ШТАТНЫМИ СРЕДСТВАМИ ПРОГРАММЫ АUDАСІТҮ® (ПРОГРАММНО-ОРИЕНТИРОВАННЫЙ ПОДХОД)
}

\section{COMPUTER UPGRADING OF AUDIO MATERIAL USING AUDACITY ${ }^{\circledR}$ SOFTWARE API (SOFTWARE-ORIENTED APPROACH)}

V. Taran

Summary. The article deals with basic problems associated with applying of computer technologies when upgrading audio material. Audacity ${ }^{\circledR}$ software environment has been selected as a program framework. Major problem areas of audio material upgrading by program means were designated. Regular modules of Audacity ${ }^{\oplus}$ software applied in case of problems associated with audio material recovery are analyzed. Based on the analysis of the selected problems and taking into account the prediction of situations arising in the process of software sound processing feasible arrangements of regular modules usage for particular calculation of the processed information were suggested. The arrangement of these modules improves the quality of the processed audio material under given situations. Taking into account the software support of the builtin programming language Nyquist, some recommendations for the use of micro-scripts and scenario programs in order to comply with the software-oriented approach are suggested.

Keywords: computer technologies for audio material upgrading, softwareoriented approach, sound processing, Audacity ${ }^{\oplus}$, regular modules Audacity ${ }^{\circledast}$, programming language Nyquist.
B условиях, когда большинство аналогового аудиоматериала получает новою жизнь в цифровой оболочке, встает вопрос о качестве преобразуемого аудиопотока. Аудиоматериалы, преобразуемые в цифровую форму с таких аудионосителей как мини кассеты, стандарт-кассеты, бобины с магнитной лентой, виниловые пластинки часто имеют различные нежелательные акустические помехи, выражаемые в щелчках, шумах и пиковых перегрузках. Причем каждая из представленных помех имеет свою спектро-частотную характеристику, что осложняет процесс качественного аналогово-цифрового преобразования аудиоматериала с указанных выше носителей. В докомпьютерную эпоху преимущественно аналоговая аудионженерия предлагала довольно скудный спектр инструментов, которые могли бы устранять подобные нежелательные арте-
Таран Василий Васильевич

К.культурологии, АНОВО «Московский международный университет»; ФГБУН «Всероссийский институт научной и технической информачии РАН» allscience@lenta.ru

Аннотация. В статье исследуются базовые вопросы, связанные с применением компьютерных технологий при очистке аудиоматериала. В качестве программной основы выбрана программная среда Audacity ${ }^{\circledast}$. Обозначены основные проблемные области очистки аудиоматериала программными средствами. Проанализированы штатные модули программы Audacity ${ }^{\circledR}$, применяемые при проблемах, связанных с восстановлением аудиоматериала. На основе анализа выбранной проблематики и с учетом прогнозирования ситуаций, возникающих в процессе программной обработки звука, приведены возможные схемы использования штатных модулей для частного просчета обрабатываемой информации, расстановка которых повышает качество обрабатываемого аудиоматериала при заданных ситуациях. С учетом программной поддержки встроенного языка программирования Nyquist приводятся некоторые рекомендации по использованию микросценариев и сценарных программ в целях соответствия программно-ориентированному подходу.

Ключевые слова: компьютерные технологии очистки аудиоматериала, программно-ориентированный подход, обработка звука, Audacity ${ }^{\circledR}$, штатные модули Audacity ${ }^{\circledast}$, язык программирования Nyquist.

факт-эффекты ${ }^{1}$. Все изменилось в эпоху компьютерной аудиоинженерии, когда стало возможным проектировать и эмулировать процессы аппаратной обработки звука на программной основе. Программная компьютерная основа расширила и качественно улучшила процессы реставрации и преобразования аудиоматериала,

Прим. автора. Под скудным спектром инструментов подразумевается примитивный арсенал средств, который в основном мог использоваться при линейном вводе звука, корректируя некоторые артефакты. К таким артефактам относилась пиковая перегрузка моно или стерео каналов, контроль над нежелательными шумами, контроль над нежелательными задержками аудиосигнала внутри технических приборных цепей (межблочная коммутация оборудования). После того как наука открыла новые возможности по преобразованию аналогового сигнала в цифровой, компьютерные системы, руководствуясь алгоритмами просчёта данных, получили возможность многократной (числовой) перезаписи аудиосигнала, с возможностью коррекции практически любых ошибок. 
добавив в них точность расчетных характеристик. Развитие компьютерной аудиоинженерии пошло по двум основным путям: это коммерческое программное обеспечение в области очистки аудиозаписей и свободнораспространяемое программное обеспечение в области очистки аудиозаписей. Мы попытаемся проанализировать и систематизировать общие процессы очистки аудиоматериала на базе свободнораспространяемого программного обеспечения, а также предложить некоторые новые подходы по обработке и очистке аудиоматериала от нежелательных помех взяв за основу (в качестве технического инструмента) свободнораспространяемую программную микросреду Audacity ${ }^{\circledR 1}$. Audacity ${ }^{\circledR}$ - это программный аудиоредактор, включающий в себя мощные (штатные) средства по устранению нежелательных помех, возникающих при аналого-цифровом преобразовании аудиосигнала с устаревших аудионосителей. В арсенале программных средств Audacity ${ }^{\circledR}$ имеются отдельные модули, обладающие графическим интерфейсом, предназначенные для борьбы с подавлением нежелательных спектральных проявлений в дизайн-образе аудиоформы. Перечислим основные программные модули: Repair ${ }^{2}$, Normalize ${ }^{3}$, Spectral edit parametric EQ ${ }^{4}$, Spectral edit shelves ${ }^{5}, \mathrm{SC}^{6}{ }^{6}$, Spectral edit multi tool ${ }^{7}$, Click

\footnotetext{
1 Прим.автора. Программный код Audacity ${ }^{\circledR}$ распространяется по регламентам второй версии общедоступной лицензии (GNU). Автором статьи был выполнен перевод оригинального текста лицензии на русский язык соответствующий современным тенденциям и реалиям развития компьютерной техники. Данное обстоятельство позволяет использовать программный код рассматриваемого в авторских исследованиях аудиоредактора на свободной основе. Перевод оригинального текста лицензии находится в приложении к научной статье. Перевод лицензии на русский язык осуществлен в целях развития культуры программирования (изменения и перераспределения машинно-программного кода). Выполняя лицензионное соглашение, программисты и исследователи в области компьютерных наук отдают дань уважения к труду первоначальных авторов рассматриваемой нами компьютерной программы.

2 Repair - инструмент восстановления спектро-акустических частотных характеристик. Обрабатывает аудиоматериал путем сопоставления частотных характеристик и впоследствии их заменой на апроксиммированные значения. ${ }^{3}$ Normalize - инструмент нормализации звукового давления. Применяется, когда необходимо выровнить уровень громкости канала.

${ }^{4}$ Spectral edit parametric EQ — инструмент спектрального редактирования аудиоформы. Позволяет уравнивать верхние, средние и нижние частоты в спектральной составляющей.

${ }_{5}^{5}$ Spectral edit shelves - инструмент восстановления аудиоматериала, использующий низкочастотные (от 0 Гц до 500 Гц при плотности в -20 Дб) и верхне-частотные (от 5000 Гц до верхней границы частоты Найквиста) фильтры. При объединении фильтров низких и высоких частот фильтр при спектральной выборке - низкие частоты 200 Гц, верхние соответствен$\mathrm{HO}-10000$ Гц.

${ }^{6}$ SC4 (stereo compressor) - модуль компрессии стерео-дорожки, применяемый в тех случаях, когда необходимо выполнить компрессию без прогнозирования уменьшения объема канала в соотношении с пиками.

Spectral edit multi tool - инструмент спектрального редактирования применяется при спектральной выборке аудиоматериала совместно с Repair для выравнивания нижних и верхних частот.
}

Removal $^{8}$, Plot Spectrum ${ }^{9}$, Find Clipping ${ }^{10}$, Clip Fix ${ }^{11}$, Noise Reduction $^{12}$, Truncate Silence ${ }^{13}$, Change Speed ${ }^{14}$. Каждый из этих модулей направлен на определенные действия, связанные с очисткой аудиодорожки от посторонних помех. Модули спроектированы таким образом, чтобы оператору было легко задавать числовые параметры и устанавливать порог отсеивания нежелательного спектрального сегмента. К сожалению, в творческо-техническом плане какого-то универсального подхода к очистке аудиоматериала от нежелательных шумов не существует. Каждый аудиоматериал индивидуален, имеет свои как технические, так и акустические свойства, которые обычно усложняют процедуры, связанные с реставрацией аудиоматериала. В большинстве случаев звукооператору приходится надеяться, прежде всего, на собственный слух и способности уловить различные акустические нюансы восстанавливаемого трека. Это несмотря на то, что цифровая реставрация аудиоматериала подразумевает визуальную ориентированность звукооператора и автоматизирует многие процессы, направленные на улучшение частотно-балансных характеристик. Более того среди специалистов по обработке звуковых данных до сих пор ведутся оживленные дискуссии, а местами споры по поводу цифрового аудиоредактирования. Дискуссии эти рождаются в результате анализа обработанного аудиоматериала с применением компьютерных технологий и обработки того же материала с применением аналоговых приборов. В результате мы видим разность взглядов на технические процессы обработки аудиоматериала. Нередко можно услышать среди не только специалистов в области аудиообработки, но и меломанов, тех людей чья профессия связана с прослушиванием конечного аудиопродукта и его оценкой, точку зрения следующего содержания. Запись,

${ }^{8}$ Click Removal - инструмент редактирования и очистки аудиоматериала от специфических звуков (кликов), возникающих при воспроизведении аудиоматериала с виниловых носителей.

${ }^{9}$ Plot Spectrum - инструмент частотного анализа аудиоматериала. Имеет разные варианты графического представления частотной амплитуды, что помогает аудиоинженеру более гибко редактировать аудиоматериал в режиме стандартной звуковой формы, а также в режимах осциллограммы и спектрограммы.

${ }^{0}$ Find Clipping - поиск пиковых значений амплитуды (обрыва) сигнала, приводящих к перегрузке канала и образующих нежелательные звуки. Инструмент удаляет нежелательные помехи (клипы), используя метод выборки по маркированным аудиоинженером значениям.

${ }^{11}$ Clip Fix - инструмент восстановления пиковых значений амплитуды аудиосигнала, использующий интерполяцию потерянных значений.

${ }^{12}$ Noise Reduction - модуль шумоподавления борется с такими негативными проявлениями в аудиосигнале, как шум, шипение, свист, гул, треск и т.д.

${ }^{13}$ Truncate Silence - модуль отсеивания тишины, возникающей между соединенными аудиофрагментами либо нежелательной тишины, спектр которой имеет минимальные амплитудные значения, проявляющие себя как нежелательный шум.

${ }^{14}$ Change Speed - модуль изменения скорости воспроизведения аудиоматериала. Используется для коррекции и выравнивания скорости оцифрованного материала. 


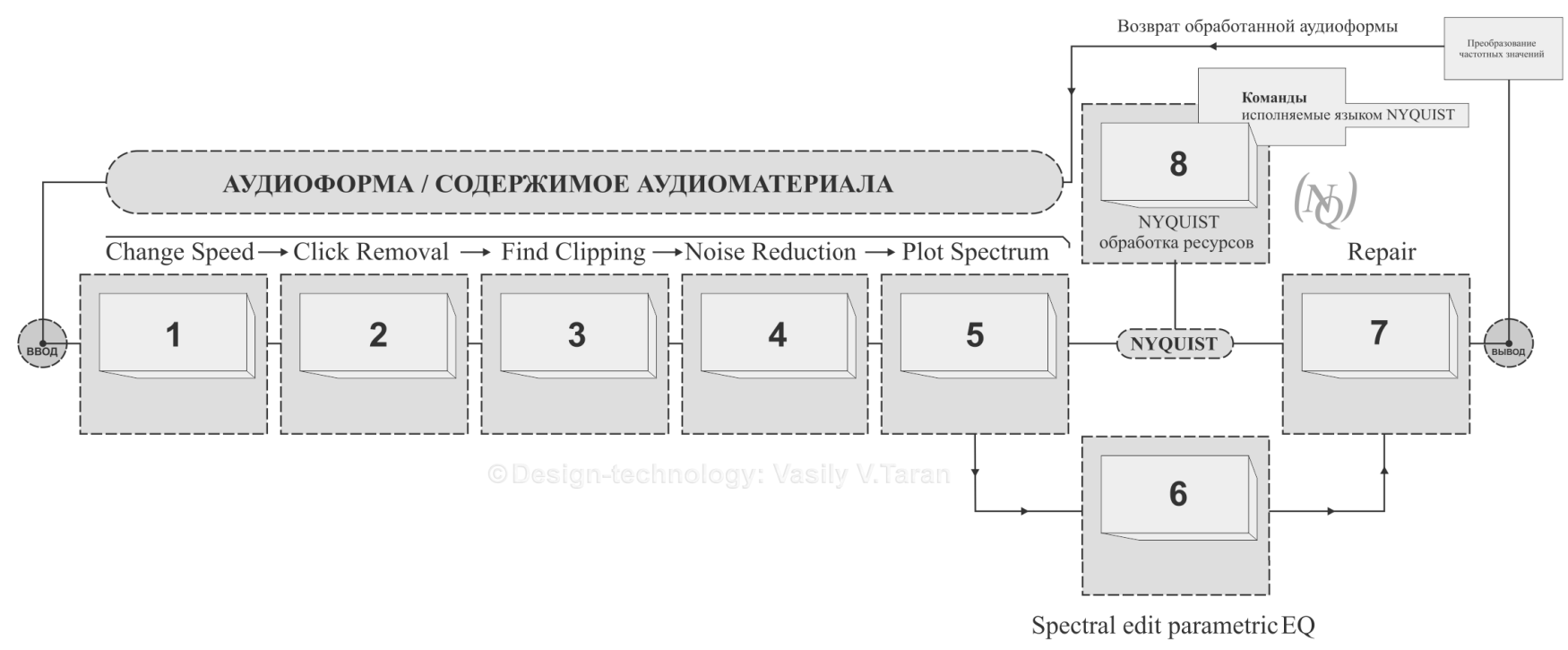

Рис. 1. Незамкнутый цикл обработки аудиоматериала интерфейсно-ориентированным способом, с возможностью корректировки производимых оператором действий на языке программирования Nyquist.

Источник: составлено автором.

проходящая процедуру аналого-цифрового преобразования для её последующей обработки и уже обработанная вычислительными возможностями компьютерной программы запись, проходящая обратную процедуру цифро-аналогового преобразования, теряет некую колоритность звучания. Причем такая точка зрения опирается исключительно на психоакустическое восприятие прослушиваемого аудиопродукта. Потому что технически воспроизводимая запись может быть очень качественной записью с характеристикой, к примеру 192000 Гц, имеющая 32-х разрядный формат. Сегодня компьютерная очистка аудиоматериала технологически имеет схожие принципы обработки в независимости от применяемого при данных процедурах программного обеспечения. Техническая суть её такова. Как правило, за основу берётся участок спектро-частотных характеристик, имеющих нежелательные шумы (электромагнитные помехи, шипение, свистящие звуки, гул привода кассетной деки и т.д.); затем компьютерная программа делает снимок нежелательного участка, который сохраняется во временной памяти. Далее оператор выполняет корректировку снятых спектро-частотных характеристик, ориентируясь на слух или плагин визуализации спектра в Audacity ${ }^{\circledR}$, под названием Plot Spectrum, затем оператор применяет частотные значения снимка к проблемным участкам обрабатываемого аудиоматериала. Программа в скрытом виде начинает производить пересчет аудиоданных в соответствии со снимком таким образом, чтобы эксплицировать из общей аудиоформы только те значения, которые заданы оператором. В случае с шу- мами типа шипения или гула алгоритм сужает динамический диапазон до предела заданного оператором ${ }^{1}$. После процедуры экспликации установленных оператором частотных значений программа пересчитывает вес аудиоформы и обновляет спектро-частотные показатели. Эту процедуру можно назвать «эффект шаблона», поскольку применяется один и тот же принцип фактически ко всей очистке аудиоматериала. Рассмотрим самые частые случаи, когда такого рода подход бывает оправдан в условиях борьбы с шумами, и проведем анализ, насколько он эффективен в профессиональной очистке аудиоматериала от сторонних помех. В процессе воспроизведения аналогового материала с целью его дальнейшей перезаписи в цифровой вид могут происходить различные ошибки, которые в конечном итоге могут снижать качество воспроизводимого аудиоматериала. Разделим эти ошибки на две группы. Группа а) — это ошибки, возникающие непосредственно при воспроизведении аудиоматериала с аудионосителя, а группа б) - это ошибки, которые возникают в процессе конвертации адиоматериала программно-аппаратным способом.

К группе а) (в зависимости от типа носителя) относятся следующие виды ошибок:

\section{Вини^овая п^астинка}

В практике компьютерного шумоподавления такой тип корректировки шумов называется компандерным (companding встречается также compansion) фактически это подавители либо расширители участка частотного диапазона. 


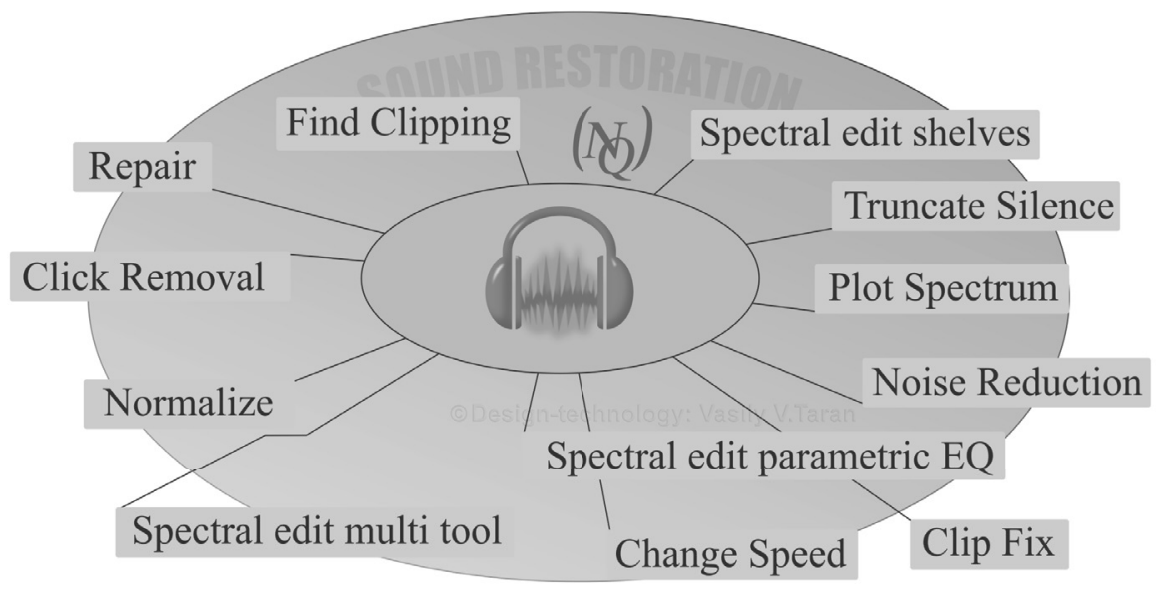

Рис. 2. Программная среда Audacity ${ }^{\circledR}$ (взаимодействующая с языком Nyquist) и её штатный модульный инструментарий, направленный на реставрацию аудиозаписей различной степени сложности.

Источник: составлено автором.

1. Проигрыватель некорректно воспроизводит скорость, установленную самим носителем.

2. Считывающее устройство (звукосниматель) загрязнено и препятствует качественному воспроизведению звука создавая нежелательные помехи.

3. Каналы пластинки имеют дефекты, видимые на глаз и невидимые повреждения.

4. Пластинка имеет дефекты в результате попадания на неё различных химических жидкостей, способных повредить каналы.

Штатные модули Audacity ${ }^{\circledR}$ способные корректировать указанные выше проблемы: Change Speed, Click Removal, Find Clipping, Noise Reduction, Plot Spectrum, Spectral edit parametric EQ, Repair.

Change Speed обычно применяется, когда появляется проблема 1). К сожалению, не все устройства одинаково точно воспроизводят аудиопоток, особенно это касается аналоговой техники. Свойства виниловых проигрывателей воспроизводить носители в соответствии категории $\mathrm{RPM}^{1}$ различались, прежде всего, в зависимости от общей схемотехники проигрывателя и от ротационного устройства в частности. Категориально было выделено несколько скоростей вращения виниловых носителей 16 rpm 33 rpm 45 rpm 78 rpm. Поэтому при оцифровке аудиоматериала с аналогового носителя (виниловая пластинка) крайне важно использовать качественное устройство воспроизведения с хорошим ротационным

' RPM (Rotation Per Minute) - аббревиатура, обозначающая скорость вращения винилового диска в минуту. Является важным индикатором при воспроизведении пластинки. Несоответствие скорости данному стандарту может привести к погрешности воспроизведения аудиокомпозиции, обычно проявляется как увеличение либо замедление воспроизводящего потока. механизмом. Конечно, в студийных условиях есть большие возможности для оцифровки подобного материала, однако существует огромное количество любителей-меломанов, которые обладают достаточно солидными комплектами виниловых носителей, которые самостоятельно оцифровывают аудиоматериал.

И в таких условиях чаще всего и возникают подобные ошибки. Инструмент Change Speed годится как раз в таких ситуациях, когда аппаратным способом в (любительских) условиях поставленную проблему решить невозможно, но запись, которая оцифровывается в таких условиях может иметь огромную ценность. Модуль Change Speed задает скорость проигрывания аудиоматериала от 0,010 до 5,000 и процентное соотношение от $-99,000$ до 400,037, например так 0,520 / -48,000. В модуле предусмотрены три значения $\mathrm{rpm}=33,45,78$ и соответствующая им длительность оцифрованной аудиоформы current length / new length (текущая длина/новая длина). Хочется отметить, что штатным объектно-ориентированным модулем Change Speed предусмотрено изменение rpm только трёх значений 33,45,78. Если пластинка нестандартная, к примеру, 16 rpm, то возможно применить программно-ориентированный подход.

Концепция программно-ориентированного подхода² (включает интерфейсно-ориентированные функции аудиоридактора) и предполагает комплексное исполь-

${ }^{2}$ Прим.автора. Не смотря на использование в инженерных целях модуля Nyquist Prompt, концепция программно-ориентированного подхода акцентирует в большей степени своё внимание на применении объектно-ориентированных модулей обработки звука, однако это не снимает с процедурной повестки те ситуации, когда необходимы сверхточные настройки алгоритмов обличённых в интерфейсную оболочку (речь идёт об аудиопректах высокой сложности). 
def i ne $r$ ot at i on $16-p 5=(n 1, p 7, k 4, n s 7, r 5$, sp5, np8, j 5),

rst $-d b 5-16=(1,7,4,7,5,5,5,8,5)$

def i ne channel $\mathrm{s} \mathrm{m} / \mathrm{s}$

pns - rq5 = I ( sf 5, d7, sk8, gx7, vc6, j। 3, h5, b6),

( sf 5, d7, sk8, gx7, vc6, j।3, h5, b6)

rst - sy5 $=r(f 6, a 7, r 3, m 8, x 9, h 3, q 7, z 5)$

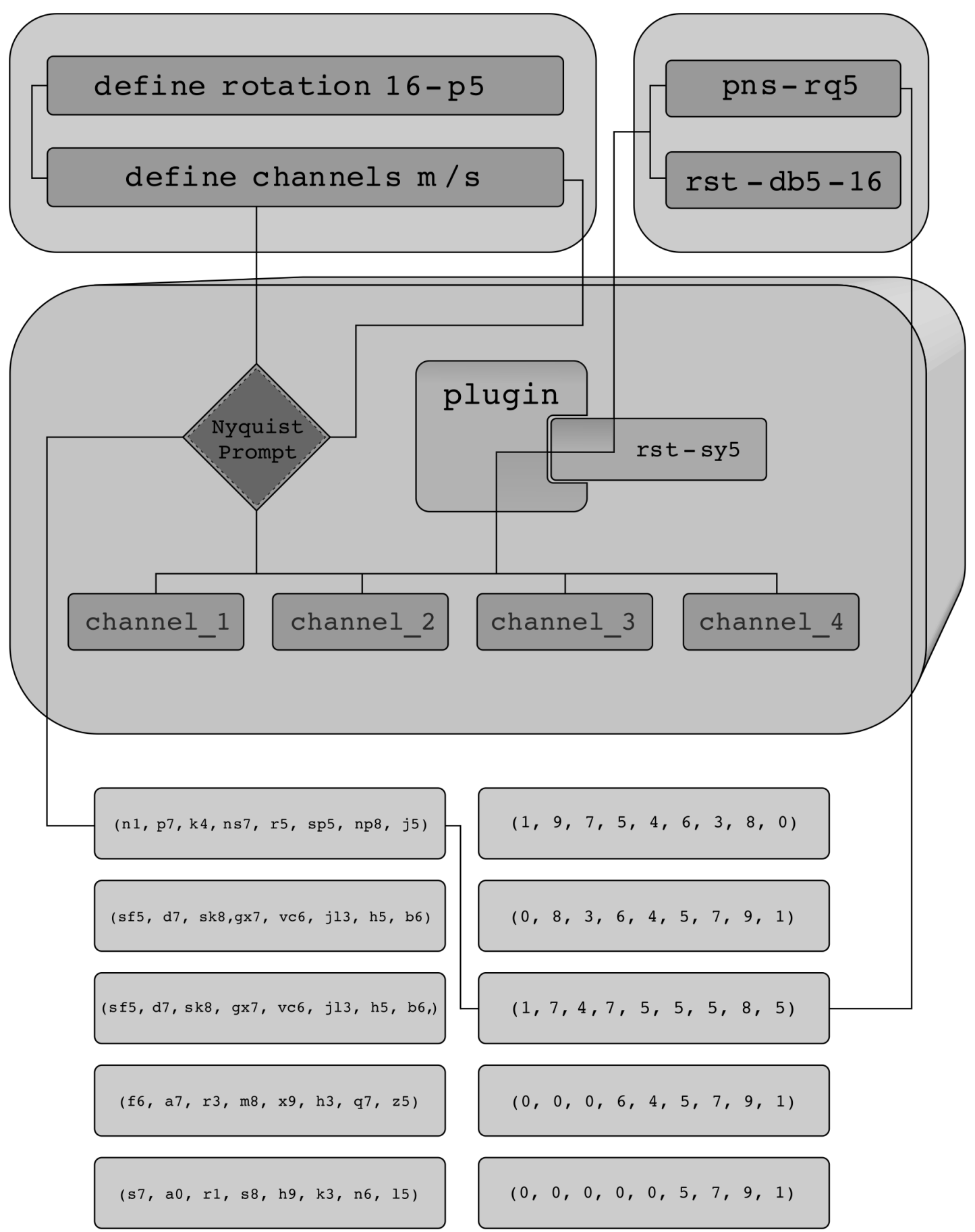

Рис. 3. Блок-схема, иллюстрирующая процессы взаимодействия программных фрагментов кода. Показывает вероятность распределения кода и соответствующих ему числовых значений при обработке аудиосигнала штатным подключаемым модулем (плагином).

Источник: составлено автором. 


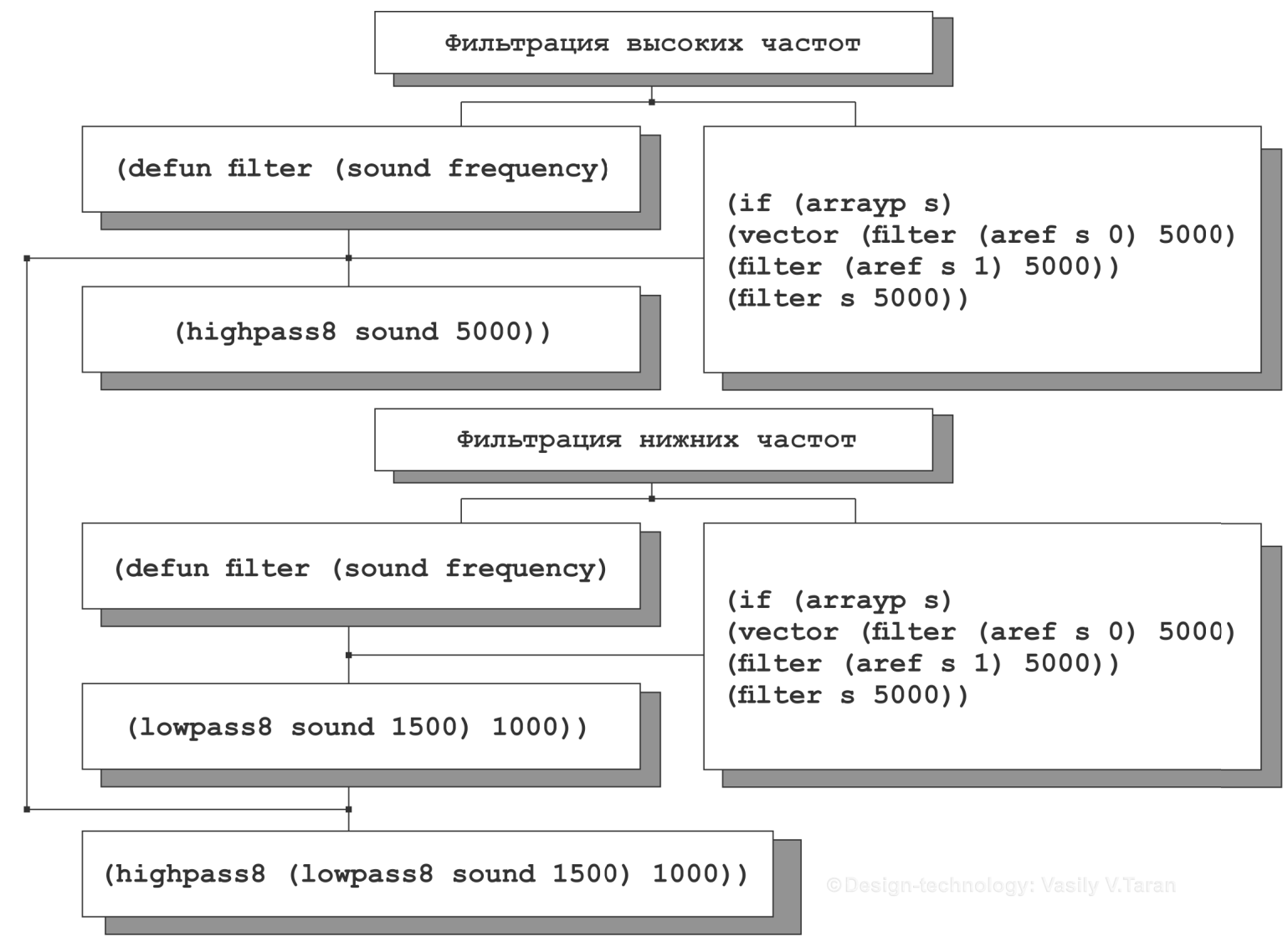

Рис. 4. Блок-схема, визуализирующая исполнение инженерного кода языка Nyquist, а также характеризующая процессы фильтрации нижних и высоких частот, в соответствии с выполняемыми процедурами.

Источник: составлено автором.

зование штатных средств программы Audacity ${ }^{\circledR}$ в комплекте с терминальным средством управления с целью обеспечения более точного редактирования аудиоматериала. Для этого необходимо воспользоваться средой выполнения команд Nyquist Prompt, которая может значительно расширить функции каждого штатного объектно-ориентированного модуля обработки аудиоданных или ввести новые функции, способные обрабатывать аудиоданные независимо от встроенных модулей. Допустим нам необходимо программно установить значения от 16 до 16 оборотов в минуту, при этом идентифицируя источники каждого из каналов моно/стерео'. Запускаем Nyquist Prompt и описываем данную процедуру² на язы-

\footnotetext{
${ }^{1}$ Необходимо помнить, что язык Nyquist работает с массивами, поэтому каналы стерео обрабатываются по раздельности, поэтому необходимо применять описанный выше фильтр к каждому из этих элементов в массиве. ${ }^{2}$ Прим.автора. При описании процедур обработки звука следует помнить о функциях языка Nyquist в частности о звуках, которые являются примитивами и включают пять компонентов: [srate —типовой уровень звука]; [samples — образцы]; [signal-start — время первого образца]; [signalstop — время прошедшего образца]; [logical-stop — время, при котором
}

ке программирования Nyquist ${ }^{3}$ с возможностью интерпретации синтаксиса XLISP4.

звук имеет логическое завершение обычно заканчивается в начале затухания]. Более подробно познакомиться с функциями языка Nyquist можно в официальном справочном руководстве Nyquist конкретно в главе 7 под названием «Функции языка Nyquist», анг. название: Nyquist Reference Manual (C) Dannenberg R. B.) - Version 3.15, Carnegie Mellon University/School of Computer Science/Pittsburgh, PA 15213, U.S.A. September 11, 2018 Chapter 7. Nyquist Functions pp 66-67.

${ }^{3}$ Прим.автора. Nyquist - язык программирования, разработанный инженером Роджером Данненбергом (Dannenberg, R. В.) с целью облегчения задач по обработке звука в программной среде Audacity ${ }^{\circledR}$, основывается на синтаксической базе XLISP и может исполнять синтаксис языка SAL. Последнее обстоятельство способствует расширению некоторых функций по составлению микропрограмм, использующих алгоритмы средней сложности. Поддержка языка SAL объясняется желанием авторов данного проекта обеспечить интеграцию алгоритмизации с интерпретацией действий заданных звукоинженером, SAL здесь подходит лучше всего, поскольку является алгоритмизированным языком.

${ }^{4}$ LISP (LISt Processing language) - алгоритмический язык программирования, направленный на решение задач начальной, средней и высокой степеней сложности в разных областях интеллектуальной деятельности. Синтаксическая структура языка Nyquist во многом схожа с диалектом XLISP. 
Схема фильтрации частот выглядит следующим образом

В представленном примере функция vector, помимо инициализации векторных значений, ещё может интегририровать массивы каналов воспроизведения L/R MONO/STEREO [1,2].

В процессе оцифровки аудиоматериала может потребоваться нештатная балансировка каналов или разграничение массивов аудиоданных. В этой ситуации полезными будут следующие операторы функции:

- (arrayp s) - возвращает true, если [s] \массив

- (aref s 0) - первый элемент в массиве [s] \левый канал

- (aref s 1) - второй элемент в массиве [s] \правый канал

- (setf s (make-array 2)) — превращает [s] \в следующий массив длины [2]

- (setf (aref s 0) left) - оставляет первый элемент массива [s]

- (setf (aref s 1) right) - делает право вторым элементом массива [s]

Click Removal, Find Clipping, Noise Reduction, Plot Spectrum, Spectral edit parametric EQ, Repair - данные модули могут успешно применяться при проблеме 2). Обратим внимание, что модуль Find Clipping при его некоторой модернизации в условиях среды Nyquist может использоваться для обнаружения ударов при производстве популярной музыки и также удалять нежелательные биты [3]. В статье Р. Даннеберга под названием «Перспективы компьютерной музыки» описываются технико-философские проблемы развития программной инженерии в данной области. В частности анализируется проблема производительности программного обеспечения в области нелинейной обработки аудиоматериала, в том числе акцент делается на язык программирования $\mathrm{C}++$ как наиболее мобильном решении в области проектирования прикладных аудиопрограмм. Однако статья опубликована в 1996 году и несколько годами позднее на основе LISP автором излагаются концепции языка Nyquist ${ }^{1}$ [4]. При загрязнении считы-

\footnotetext{
Проблематика разработки компьютерных программ, утилит и небольших приложений в сфере аудиоиндустрии является дискуссионной. В первую очередь, это касается выбора языка программирования для разработки подобных компьютерных приложений, способного в равной мере удовлетворять актуальные времени потребности прикладного характера, и очистка аудиоматериала от шумов здесь не является исключением (с точки зрения звукорежиссёра, инженера сведения, аудиоинженера, технического ассистента и т.д.) и отвечать запросам требовательности к аппаратным ресурсам, системной совместимости (с точки зрения программистов, специалистов в области компьютерных наук, прикладной информатики). Таким образом, необходимы такие решения в области программирования, которые были бы адекватными, функциональными и простыми для понимания с точки зрения технических команд (на уровне звукорежиссёра, когда необходимо решить какую-либо утилитарную задачу, а интерфейс программы не в состоянии
}

вающего устройства могут возникать нежелательные помехи разного рода. Вид помех будет зависеть от типа загрязненности считывающего устройства. Например если считывающее устройство деформировано вследствие механического повреждения то в этом случае наблюдается эффект треска либо эффект гула а также в некоторых случаях эффект статического электричества. В этом случае необходимо воспользоваться штатным модулем Plot Spectrum, чтобы произвести частотный анализ аудиоматериала с целью сепарирования нежелательных шумов. После сепарации, когда нежелательные пиковые значения будут оформлены соответствующими им частотами можно задействовать остальные штатные инструменты для устранения помех в зависимости от их типа. Click Removal и Find Clipping здесь могут выступить в роли подавителя скачков статического электричества ${ }^{2}$. Noise Reduction пригодиться если в оцифрованном аудиоматериале присутствует шип, гул и шум. А инструменты Spectral edit parametric EQ и Repair послужат хорошими средствами восстановления аудиоформы после примененных процедур декликинга ${ }^{3}$ и деклиппинга ${ }^{4}$, а также шумоподавления. При проблемах, возникающих при обработке аудиоматериала 3) и 4). Когда пластинка имеет механические дефекты каналов либо дефекты тех же каналов в результате попадания на неё различных разъедающих поливинилхлорид ${ }^{5}$ химических веществ, де-

удовлетворить предъявляемые требования) и в то же время эти же команды входили бы в компилируемую оболочку самого языка, на котором изначально изложен оригинальный код программы. Язык Найквист (в англоязычной научной литературе в области компьютерных наук: Nyquist programming language coкр. Nyquist PL, в некоторых текстах Nyquist ${ }^{\mathrm{PL}}$ ), спроектированный американским инженером Роджером Данненбергом (Roger B. Dannenberg), является попыткой создания языка такого типа, о котором говорится выше в нашем примечании. В этом отношении очень интересной с теоретико-прикладной точки зрения представляется статья под названием «Среда композиции языка Найквист, поддерживающая текстовое программирование задачами, ориентированными на пользовательский интерфейс» в оригинале опубликованная: Roger B. Dannenberg, «The Nyquist Composition Environment: Supporting Textual Programming with a Task-Oriented User Interface», in Proceedings of the 2008 International Computer Music Conference, San Francisco, CA: The International Computer Music Association, August 2008. @ 2008, Roger B. Dannenberg.

${ }^{2}$ Акустический спектр эффекта статического электричества во многом схож с обычным треском, возникающим при неправильной работе механики воспроизводящего звуковой поток устройства. Поэтому к цепочке Click Removal и Find Clipping можно добавить модуль Noise Reduction.

${ }^{3}$ Прим.автора. Декликинг - процесс удаления нежелательных частот, выражающихся в акустических погрешностях аудиосигнала. Данный эффект встречается в основном при оцифровке аналоговых носителей (аудиокассет, ленточных бабин, виниловых пластинок), которые подвергались размагничиванию или намагничиванию либо в случае с винилом - искажение (загрязнение) воспроизводящих информацию дорожек пластинки.

${ }^{4}$ Прим.автора. Деклиппинг — процесс искусственного предотвращения перегрузок различных частот аудиоматериала путем обрезания нежелательных частот. Применяется при сведении дорожек имеющих разную степень плотности звучания для подгонки частотных характеристик. Также процесс нашел своё применение при финализации аудиоматериала, его применение в этой области ограничивается срезом уплотнения частот на каналах аудио.

5 Поливинилхлорид полимер, который применяется при изготовлении виниловых пластинок. Из-за того, что сам поливинилхлорид имеет высокую степень 


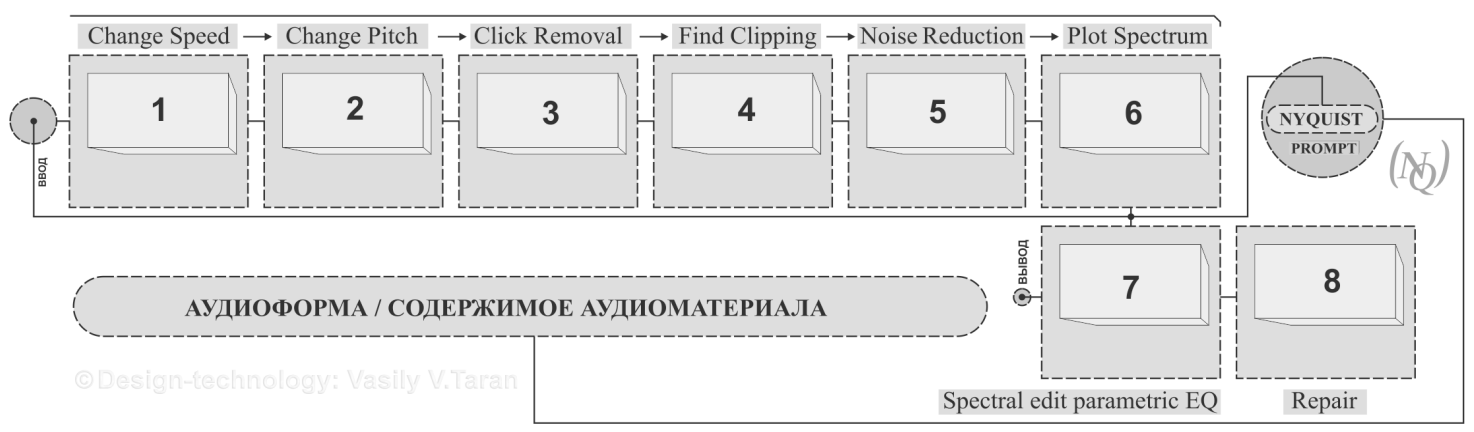

Рис. 5. Незамкнутый цикл обработки аудиоматериала с вариантом поэтапного применения штатных подключаемых модулей Audacity ${ }^{\circledR}$.

Источник: составлено автором.
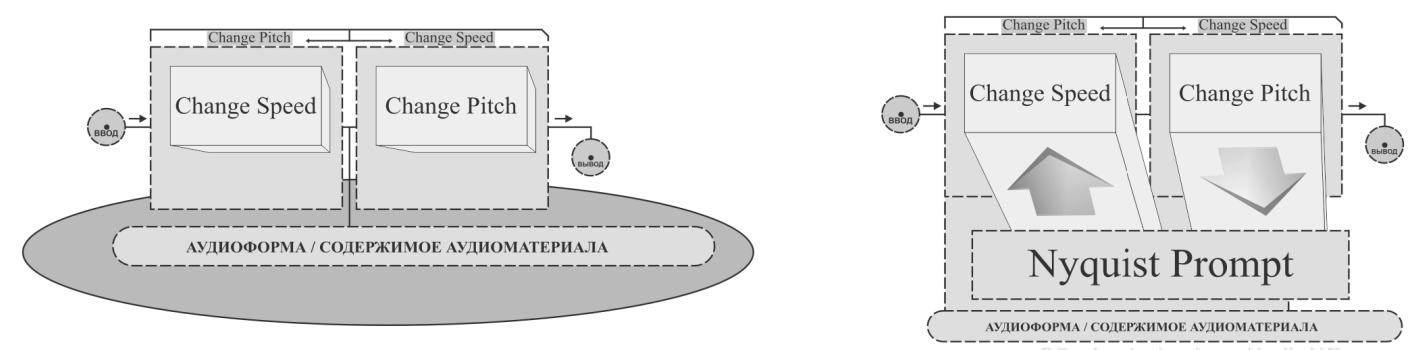

Рис. 6. Варианты использования связки плагинов Change Speed и Change Pitch.

а. Рисунок слева показывает использование модулей интерфейсно-ориентированным способом.

b. Рисунок слева показывает возможности виртуальной маршрутизации обрабатываемого аудиосигнала через Nyquist Prompt.

Источник: составлено автором.

фекты при воспроизведении аудиоматериала могут выражаться в глухости звучания аудиоматериала, в плохо читаемых фрагментах аудиопроизведения, сбивчивости ритма воспроизведения, резкого повышения громкости, либо сползания считывающего устройства на соседние каналы (эффект пилы). В этой ситуации к перечисленным выше штатным модулям Click Removal, Find Clipping, Noise Reduction, Plot Spectrum, Spectral edit parametric EQ, Repair, имеющим отношение к проблеме 2), прибавляются ещё два модуля Normalize и SC4. Дело в том, что при моделируемой ситуации процессы поведения считывающего устройства оказываются предсказуемы и выдают схожие эффекты, описанные в проблеме 2). Кроме резкого повышения громкости и эффекта пилы. В случае с повышением громкости на поврежденных участках канала виниловой пластинки важно использовать мо-

изнашиваемости к нему добавляются сополимеры винилхлорида и винилацетата в особой пропорции. Помимо сополимеров для обеспечения упругости пластинки добавляются различные пластмассостойкие химические вещества. дуль Normalize в паре с SC4. Модуль Normalize призван нормализовать амплитуду звукового давления как на отдельных участках аудиоформы, так и на всей аудиоформе а модуль SC4 сможет гибко выполнить компрессию, соблюдая баланс между $\mathrm{RMS}^{1}$ и пиками.

Аудиокассета, магнитофонная магнитная лента

1. Проигрыватель некорректно выполняет ротацию магнитной ленты.

2. Считывающее устройство загрязнено, создает помехи и препятствует качественному воспроизведению.

3. Считывающее устройство сильно намагничено, в результате чего при воспроизведении может появиться гул.

RMS (Root Mean Square) — корневое среднеквадратическое значение. Специальный метод, который позволяет вычислить математическое значение для среднего уровня плотности аудиоформы. 


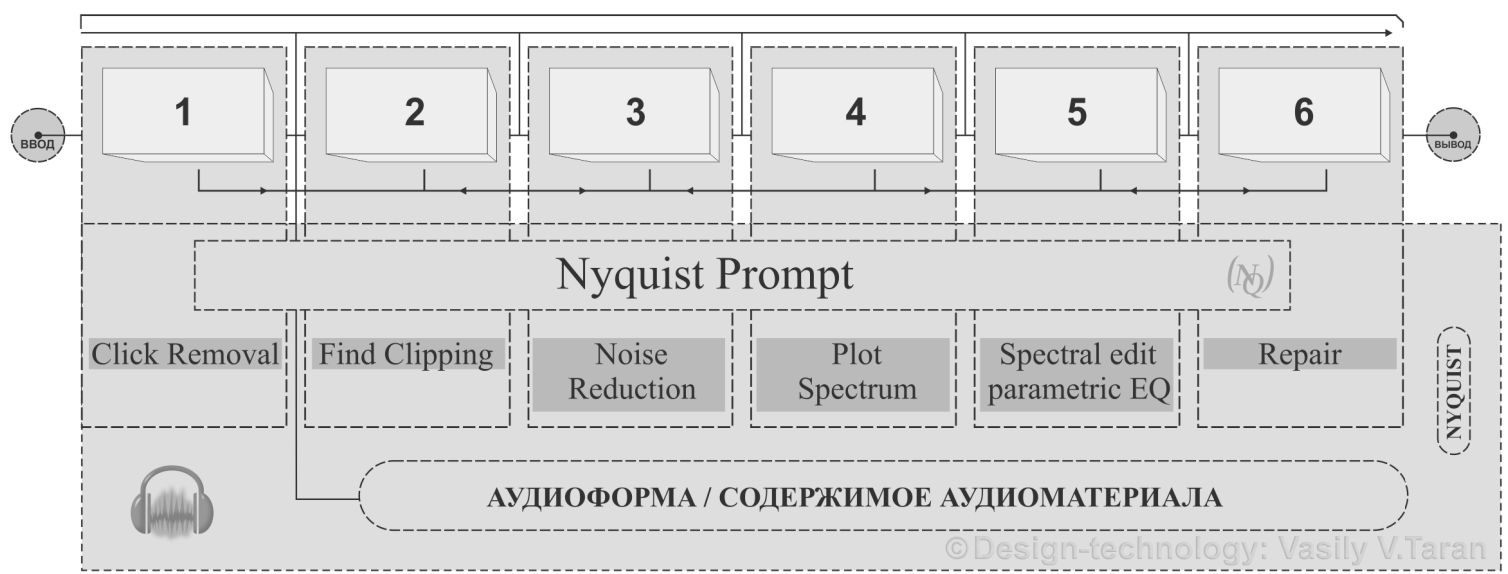

Рис. 7. Циркулярный тип обработки аудиоматериала, представляющий замкнутые циклы обработки аудиоданных на отдельных участках цепи, при этом вывод стереоканала обрабатываемой аудиоформы имеет незамкнутый цикл.

Источник: составлено автором.

4. Потеря акустических свойств магнитной ленты в результате её размагничивания либо осыпания.

Штатные модули Audacity ${ }^{\circledR}$, способные корректировать указанные выше проблемы: Change Speed, Change Pitch, Click Removal, Find Clipping, Noise Reduction, Plot Spectrum, Spectral edit parametric EQ, Repair.

В первом случае 1), когда ротационные механизмы проигрывателя некорректно вращают бобину магнитной ленты, особенно полезными оказываются штатные модули Change Speed, Change Pitch.

Change Speed - подгоняет скорость вращения (обороты вращения) под стандарты аудиокассеты. Change Pitch - применяется в нескольких случаях:

- Если во время фазы вращения на некоторых участках ленты падает скорость оборотов.

- Если магнитная лента перезаписана с виниловой пластинки и при перезаписи аудиоматериала скорость воспроизведения оригинального носителя была искажена, а впоследствии, при воспроизведении с магнитофона, был нарушен воспроизводимый масштаб времени.

- Если необходимо подкорректировать (растянуть) фрагментарные акустические участки, не изменяя частоты воспроизведения.

Click Removal, Find Clipping, Noise Reduction, Plot Spectrum необходимы в ситуации 2), 3). При загрязнении и намагничивании считывающего устройства появляются шумы разного спектра, бороться с ними можно с помощью указанных модулей. И в положении 4), когда магнитная лента теряет свои акустические свойства, на помощь приходят специальные модули Plot Spectrum, Spectral edit parametric EQ, Repair. Plot Spectrum (в этой ситуации) используется как инструмент точного частотного анализа для определения акустических нюансов. Например, при деформации пленки может возникнуть эффект заглушения аудиоматериала. С помощью данного модуля можно выявить его частотные характеристики, сохранить их математические значения и при повторении подобных дефектов использовать как шаблон. Spectral edit parametric EQ - позволит выравнивать спектральную составляющую аудиоматериала как на отдельных участках так и на всем материале.

Repair - позволит исправить спектральные характеристики в корреляции с общим спектром.

Компакт-диск

1. Диск имеет физические повреждения и читается с ошибками.

2. Диск плохо воспроизводится в результате повреждения портируемой файловой системы.

3. Матрица для тиражирования компакт-дисков изначально содержала ошибку в последствии тиражируемую на другие диски.

4. В случае с использованием в профессиональной деятельности дисков, имеющих формат CD-RW, плохо выполнена финализация ${ }^{1}$ диска.

Прим.автора. Финализация - процесс закрытия перезаписываемого CD-носителя класса RW, обеспечивающий устойчивое воспроизведение компакт диска на всех проигрывателях, поддерживающих на чтение данный формат. Не путать с акустической финализацией процессом приведения различных спектро-акустических значений к заданным значениям с целью обеспечить одинаковое звучание всех частот на различных устройствах, имеющих разные атрибуты воспроизведения звука. 


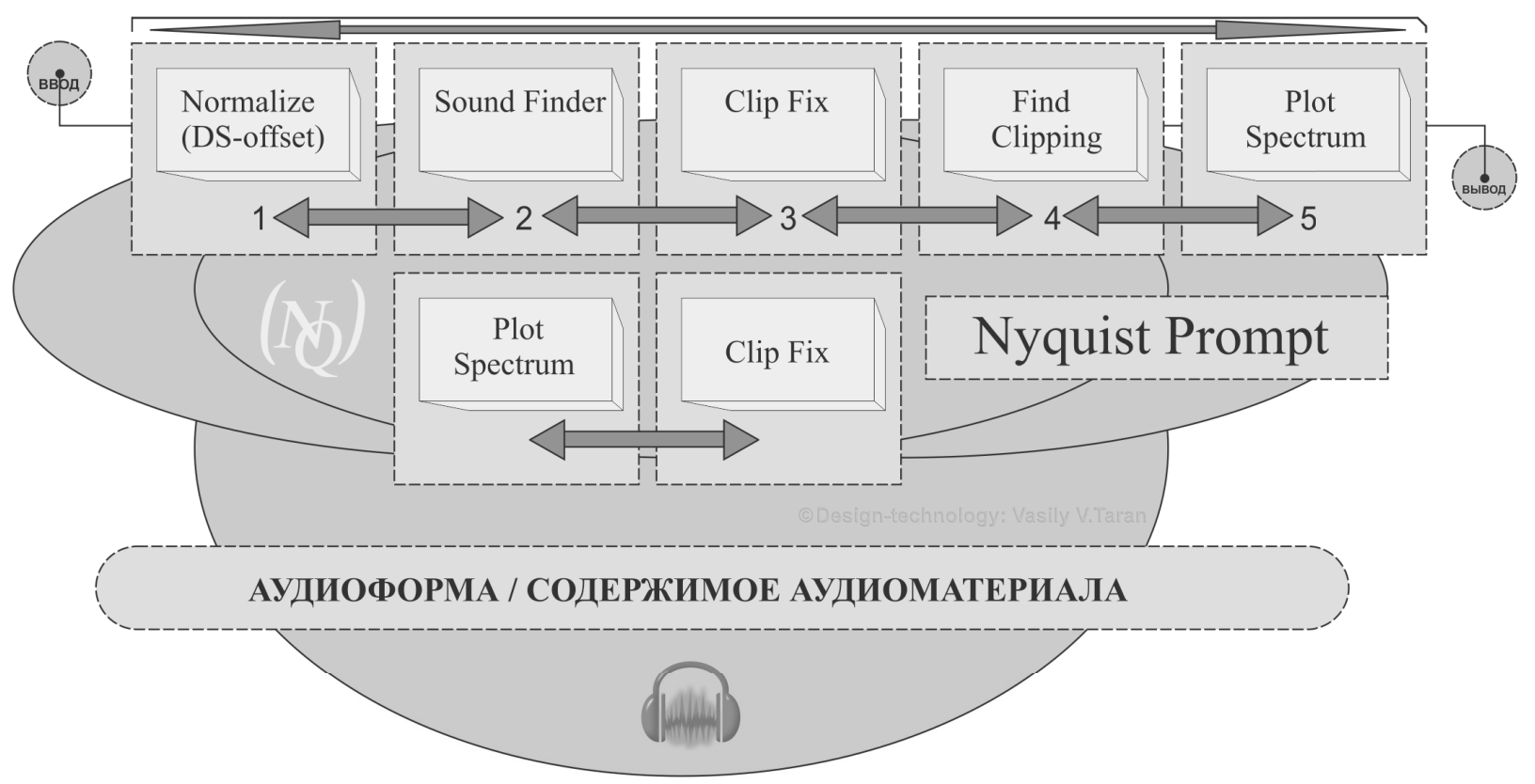

Рис. 8. Вариант использования штатных модулей обработки аудиоматериала в Audacity ${ }^{\circledast}$ пи ошибках группы б. Стрелками показана возможность попарного переключения модулей при обработке однофазного и двухфазного аудиосодержимого. Источник: составлено автором.

Штатные модули Audacity ${ }^{\circledR}$ способные корректировать указанные выше проблемы: Plot Spectrum, Sample Data Import.

В ситуации с компакт-диском практически во всех случаях, описанных положениями 1), 2), 3), 4) - основными инструментами являются Plot Spectrum и Sample Data Import. Plot Spectrum - позволяет выполнить частотный анализ нечитаемых участков, но присутствующих в аудиоформе. Sample Data Import - применяется в основном для точности воспроизведения аудиоформы при ошибках чтения, связанных с нестабильностью файловой системы либо механическими недостатками привода устройства чтения дисков. Sample Data Import это типовой импорт данных, основанный на технологии описания аудиоформы математическими значениями в текстовом файле. Корректнее всего работает с кодировкой ASCII при манипуляции с языком Найквист (Nyquist), возможно воспроизведение и в других кодировках. Создает РСМ-образцы ${ }^{1}$ (привязки) для каждого числового значения. Читает 32-х разрядные значения (значения с плавающей запятой) в диапазоне +/-1.0 выражает нулевую амплитуду. Текстовой файл должен иметь расширение

1 РСМ-образцы - образцы импульсно-кодовой модуляции. Импульсно-кодовая модуляция (Pulse Code Modulation) - специальный метод записи аудиоданных, данный принцип записи лежит в основе многих аудиофайлов. Ярким примером подобной записи в аудиоформат является формат WAVE, разработанный корпорацией Microsoft. (.txt) и придерживаться жесткой структуры - все значения должны отделяться пробелами либо разрывами строки между значениями. При работе с аудиоматериалом, имеющим деления на каналы стерео, при импорте математических значений для преобразования в аудиодорожку, изначально программе необходимо задать стерео-режим, в противном случае программа будет обрабатывать аудиоматериал в моно-режиме.

Группа б) - содержит следующий набор ошибок:

1. Отклонение конвертируемого аудиоматериала от центральной фазы в независимости от типа канального воспроизведения (моно, стерео).

2. Задержки аудиосигнала, возникающие при неправильной коммутации аудиооборудывания.

3. Изменение частоты дискретизации при автоматической передискретизации аудиоматериала.

4. Перегрузка адиоматериала пиковыми значениями, вызывающими эффект клиппинга.

В этих условиях могут быть использованы следующие штатные средства: Normalize (DS-offset), Sound Finder, Clip Fix, Find Clipping, Plot Spectrum.

Процедуры конвертации аудиоматериала могут, приводить к снижению его качества. В принципе хорошим тоном считается поступательное снижение частоты дискретизации при процедурах его конвертации, 

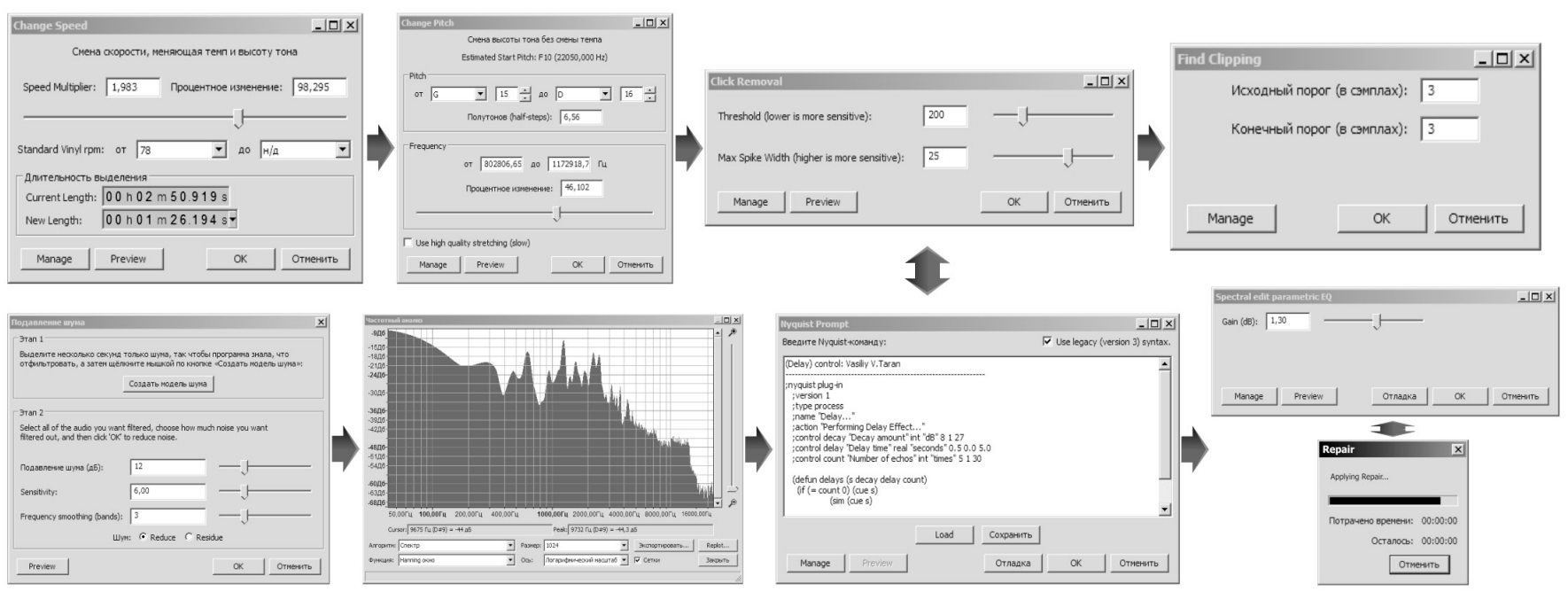

Рис. 9. Цепь интерфейсно-ориентированных модулей обработки звука между собой (применяемых попеременно), с программно-ориентированным контролем Nyquist Prompt.

т.е. от большего к меньшему. Например, оригинальный инструментальный или вокальный (живой) аудиопоток лучше записывать с большей частотой дискретизации 96000 Гц и уже потом его можно адаптировать под более низкую частоту 44100 Гц ${ }^{1}$. Конечно в большинстве случаев звукоинженер работает с уже готовым аудиоматериалом, и в этом случае приходится задавать значения частоты дискретизации искусственно. Получается задаваемая частота дискретизации является псевдо частотой, что сказывается на качестве воспроизводимого аудиоматериала.

При передискретизации аудиоматериала, в результате несовпадения массивов данных, может образоваться отклонение от центра основной фазы, несущей аудиоматериал. Ликвидировать такую погрешность поможет модуль Normalize и его встроенная функция (DS-offset). DS-offset приводит к равномерному распределению фаз аудиодорожек и сводит к минимуму отклонение от центральной фазы². В некоторых (особых) случаях, когда необходимо снять участки помех между фрагментами аудиоматериала, можно использовать Sound Finder. Модуль в автоматическом режиме в соответствии с установленными операто-

\footnotetext{
1 Частота 44100 Гц является стандартом в области цифровой звукозаписи. Она определяет средние показатели восприимчивости человеческим слухом всех основных акустических нюансов. Внедрена как стандарт для CD-носителей.

2 Отклонение от центральной фазы содержимого аудиодорожки может вызвать нежелательные (искусственные) шумы, а при передискретизации может наблюдаться потеря полезного аудиоматериала. Если материл воспроизводится в моно-режиме, потери практически не слышны. Если в стерео-режиме, возможно «наплывание» фаз одной на другую. Акустическая картина в данном случае может быть сильно деформирована.
}

ром метками устранит подобные проблемы. Помимо проблем 1) и 3) остаются нерешенными проблемы 2) и 4). Вернемся к проблеме 2), а именно к задержке аудиоматериала при его оцифровке. Чтобы оцифровать материал как студийно, так и внестудийно могут использоваться совершенно разные комплекты оборудования. Естественно чисто аппаратная обработка дает более высокие результаты с точки зрения качества аудиоматериала. Однако на практике часто используются более бюджетные решения, основанные на программно-аппаратном подходе. Если брать независимые небольшие коммерческие студии звукозаписи, то при их проектировании всегда возникает проблема соотношения цены и качества, т.е. какое оборудование ставить. Мы уже не говорим о домашних полупрофессиональных и любительских студиях, где бюджет является весьма скромным. К тому же программное обеспечение тоже стоит денег. Поэтому в данном случае программный комплекс Audacity ${ }^{\circledR}$ становится здесь незаменим. При подключении аппаратных средств Audacity ${ }^{\circledR}$ одинаково хорошо распознает драйвера этих средств, к тому же кроссплатформенность Audacity ${ }^{\circledR}$ играет на руку звукоинженерам использующим рабочие станции под управлением UNIX-подобных операционных систем. При выполнении передескритизации либо выполнении преобразования частоты дискретизации на отдельных проектах Audacity ${ }^{\circledR}$, программа использует специальную очень качественную библиотеку libsoxr, которая позволяет выполнять данные процедуры с минимальными потерями даже при резком понижении разрядности аудиоматериала, а библиотека PortAudio отвечает за качество воспроизводимого внутри программы аудиоматериала. Благодаря этому, задержки аудио- 
сигнала, возникающие при аналого-цифровом преобразовании, сводятся к минимуму. Естественно они возможны, но уже в результате грубых ошибок ${ }^{1}$ при коммутации и маршрутизации аудиосигнала. И в таких ситуациях возможно применение модуля Clip Fix в сочетании с Plot Spectrum. Clip Fix это модуль противоположный модулю Find Clipping. Основная его задача сводится к восстановлению отсеченных участков аудиосигнала за счёт интерполяции потерянного сигнала. Модуль Plot Spectrum здесь является хорошим подспорьем и позволяет производить тонкий анализ, насколько же аудиосигнал нуждается в интерполяции. Ну и соответственно эффект клиппинга, возникающий в результате перегрузок на каналах аудио, может быть устранён модулем Find Clipping.

Таким образом, мы рассмотрели различные возможности штатных средств, предусмотренных программным обеспечением Audacity ${ }^{\circledR}$, по очистке аудиоматериала от нежелательных помех. Проанализировали наиболее проблемные места восстановления аудиоматенриала. Систематизировали наиболее вероятные ошибки, являющиеся препятствием для качественного аудиопроизводства. В целом, как следует из анализа, произведенного в статье, авторская позиция придерживается программно-ориентированного подхода, когда сочетаются объектно-ориентированные возможности штатных средств с гибкой их доработкой на программной основе.

Конечно, формат статьи не позволяет выделить все технические проблемы, возникающие в подобных ситуациях. Однако поскольку данная тематика является одним из центральных вопросов компьютерной аудиоинженерии, проведенный в статье анализ и сформулированные предложения по использованию программных технических средств будут полезны для приращения научно-технического знания в области аудиоинформатики. Это даст возможность по новому взглянуть на процессы реставрации аудиоматериала на открытой программной основе и будет способствовать дальнейшему развитию дискуссий в этом направлении.

\section{Приложение}

\section{ОБЩЕДОСТУПНАЯ ЛИЦЕНЗИЯ GNU Версия 2. 1991 г., июнь².}

\footnotetext{
1 Такой ошибкой может быть неправильное поведение трансформатора фантомного питания предуселителя аудиосигнала, выдаваемое помехи и образующее задержки.

${ }^{2}$ Прим.автора. Текст лицензии опубликован по адресу: https://www.gnu.org/ licenses/old-licenses/gpl-2.0.en.html. Адрес Фонда свободного программного обеспечения (Free Software Foundation, FSF): https://www.fsf.org/.
}

Перевод текста лицензии на русский язык и использованная техническая терминология ${ }^{3}$ :Таран Василий Васильевич ${ }^{4} *$

(c) Free Software Foundation, Inc., 1989, 1991.

02110-1301, США, шт. Массачусетс, г. Бостон, Франклин Стрит 51, 5 этаж. Всем разрешается воспроизводить и распространять точные копии настоящего лицензионного документа, но изменение его не допускается.

\section{Прехисловие}

Лицензии на большинство компьютерных программ предназначены для того, чтобы не разрешать вам делиться ими и изменять их. В отличие от этого, Общедоступная лицензия GNU предназначена для того, чтобы гарантировать свободу делиться и изменять свободное программное обеспечение, то есть, чтобы убедиться, что оно свободно для всех его пользователей. Эта общедоступная лицензия распространяется на большую часть программного обеспечения Фонда свободного программного обеспечения (Free Software Foundation) и на любую другую программу, авторы которой обязуются её использовать. (Некоторые другие программы Фонда свободного программного обеспечения вместо этого обеспечиваются Общедоступной лицензией библиотеки GNU — GNU Library General Public License) (далее, Общедоступная лицензия). Вы также можете применить ее к своим программам.

Когда мы говорим о свободном программном обеспечении, имеется в виду именно свободное пользование, а не ценовой контекст. Наши общедоступные лицензии предназначены для того, чтобы вы могли свободно распространять копии данного программного обеспечения (и, если хотите, взимать плату за эту услугу), получать исходный код, изменять программное обеспечение или использовать его части в новых бесплатных программах; и чтобы вы знали, что вы можете делать эти вещи.

\footnotetext{
${ }^{3}$ Автор перевода старался использовать наиболее понятную техническую терминологию. При переводе автор придерживался, прежде всего, смысловой нагрузки и руководствовался нормами русского языка. Некоторые фразы, обороты и положения приходилось трактовать дословно.

${ }^{4}$ * Таран Василий Васильевич, специалист в области компьютерного проектирования аудиоданных разной степени сложности (включая композиционные особенности звука, инженерную обработку аудиоматериала и компьютерный аудиосинтез). Кандидат культурологии, усовершенствовал методы культурологического анализа в области комплексной оценки информационно-коммуникационных технологий, компьютерных наук и интернет-телевидения. Научно обосновал необходимость учёта культурологических факторов при рейтинговой оценке сетевой готовности стран мира. Имеет научные публикации в области компьютерного графического моделирования и проектирования. В настоящее время докторант (на соискание учёной степени доктора технических наук) ФГБУН ВИНИТИ РАН.
} 
Чтобы защитить ваши права, мы должны ввести ограничения, которые запрещают кому-либо отказывать вам в этих правах или просить вас отказаться от этих прав. Эти ограничения влекут за собой определенные обязательства для вас, если вы распространяете копии программного обеспечения или изменяете его.

Например, если вы распространяете копии такой программы безвозмездно или за плату, вы должны предоставить получателям все права, которые у вас есть. Вы должны убедиться, что они тоже получают или могут получить исходный код. И вы должны показать им эти условия, чтобы они знали свои права.

Мы защищаем ваши права посредством двух действий: (1) защищаем авторское право на программное обеспечение и (2) предлагаем вам рассматриваемую лицензию, которая дает вам законное разрешение на копирование, распространение и/или модификацию программного обеспечения.

Кроме того, для защиты каждого автора и нашей компании, мы хотим убедиться, что все осознают, что нет никакой стабильной гарантии для этого свободного программного обеспечения. Если программное обеспечение модифицировано кем-то другим и передано дальше, мы хотим, чтобы его получатели знали, что продукт, которым они обладают, не является оригиналом, так что любые проблемы, созданные другими лицами, не отразятся на репутации первоначальных авторов.

Наконец, для любой свободной программы постоянно возникают проблемы, связанные с патентами. Мы хотим избежать опасности того, что распространители свободной программы будут индивидуально получать патентные лицензии, фактически делая программу своей собственностью. Чтобы предотвратить это, мы четко заявляем, что любой патент должен быть лицензирован для свободного использования всеми или не лицензирован вообще. Ниже приводятся точные условия копирования, распространения и модификации.

\section{ОБЩЕДОСТУПНАЯ ЛИЦЕНЗИЯ GNU УСЛОВИЯ КОПИРОВАНИЯ, РАСПРОСТРАНЕНИЯ И ВНОСИМЫХ ИЗМЕНЕНИЙ}

0. Настоящая лицензия распространяется на любую программу или иную разработку, содержащую размещенное правообладателем уведомление о том, что оно может быть распространено в соответствии с условиями настоящей Общедоступной лицензии. Программа, приведенная ниже, относится к любой такой программе или разработке, а «разработка, основанная на Программе» означает либо Программу, либо любую производную разработку в соответствии с законом об авторском праве: то есть разработка, содержащая Программу или её часть, либо дословно, либо с изменениями и/или переведенное на другой язык (далее, перевод без ограничений включается в термин «модификация»). К каждому лицензиату используется обращение «You».

Деятельность, отличная от копирования, распространения и изменения, не подпадает под действие настоящей лицензии; она выходит за ее рамки. Акт запуска Программы не ограничен, а выход из Программы подпадает под лицензию только в том случае, если его содержание представляет собой работу, основанную на программе (независимо от того, была ли она выполнена путем запуска Программы). Правда ли это, зависит от того, что выполняет Программа.

1. Вы можете копировать и распространять точные копии исходного кода Программы, по мере его получения на любом носителе при условии, что вы открыто и надлежащим образом публикуете на каждой копии соответствующее уведомление об авторских правах и отказе от гарантий; сохраняете в неприкосновенности все уведомления, относящиеся к настоящей лицензии и отсутствию каких-либо гарантий; и передаете любым другим получателям Программы копию настоящей лицензии вместе с Программой.

Вы можете взимать плату за физический акт передачи копии, и вы можете по своему выбору предложить гарантийную защиту в обмен на плату.

2. Вы можете изменять свою копию или копии Программы или любую её часть, формируя таким образом разработку на основе Программы, а также копировать и распространять такие изменения или разработки в соответствии с условиями Раздела 1 выше, при условии, что вы также удовлетворяете всем этим требованиям:

а. Вы должны внести в измененные файлы четкие уведомления о том, что именно вы изменили эти файлы и фиксировать дату любого изменения.

b. Вы должны обеспечить, чтобы любая разработка, которую вы распространяете или публикуете, которая полностью или частично основана на Программе или является производной от нее или любой ее части, была лицензирована бесплатно для всех третьих лиц в соответствии с условиями настоящей лицензии.

с. Если измененная программа при запуске обычно читает команды в интерактивном режиме, вы должны дать ей команду (когда она запущена для такого интерактивного использования самым обычным способом), напечатать или отобразить объявление, включающее соответствующее уведомление об авторских правах и уведомление о том, что нет никакой гарантии (или же о том, что вы предоставляете гарантию) и что пользователи могут распространять программу в соответствии с этими условиями, 
а также сообщить пользователю, как просмотреть копию этой лицензии. (Исключение: если сама Программа интерактивна, но обычно не печатает такое объявление, для вашей разработки, основанной на Программе, не требуется печатать соответствующее объявление).

Эти требования распространяются на выполненную разработку в целом. Если определяемые разделы этой разработки не являются производными от Программы и могут надлежащим образом рассматриваться как самостоятельные и отдельные разработки сами по себе, то настоящая лицензия и ее условия не применяются к этим разделам, когда вы распространяете их как отдельные разработки. Но когда вы распространяете одни и те же разделы как часть целого, которое является разработкой, основанной на Программе, распространение целого должно осуществляться на условиях настоящей лицензии, разрешения которой для других лицензиатов распространяются на все целое, а, следовательно, и на каждую часть независимо от того, кто её написал.

Таким образом, цель данного раздела не состоит в том, чтобы претендовать на ваши права или оспаривать ваши права на разработку, выполненную полностью вами; скорее, цель состоит в том, чтобы осуществлять право контролировать распространение персональных или коллективных разработок, основанных на Программе.

Кроме того, простое объединение другой разработки (не основанной на Программе) с Программой (или с разработкой, основанной на ней) на томе носителя хранения или распространения не приводит к тому, что другая разработка подпадает под действие настоящей лицензии.

3. Вы можете копировать и распространять Программу (или разработку на ее основе в соответствии с Разделом 2) в объектном коде или в выполняемой форме в соответствии с условиями вышеизложенных Разделов 1 и 2 при условии, что вы также выполните одно из следующих действий:

а. Сопроводить его полным соответствующим машиночитаемым исходным кодом, который должен распространяться в соответствии с условиями вышеизложенных Разделов 1 и 2 на носителе, обычно используемом для обмена программным обеспечением; или,

b. Сопроводить его записанным предложением, действительным не менее трех лет, предоставить любому третьему лицу за плату, не превышающую ваши затраты на физическое распространение исходного кода, полную машиночитаемую копию соответствующего исходного кода, подлежащую распространению в соответствии с условиями вышеизложенных Разделов 1 и 2 на носителе, обычно используемом для обмена программным обеспечением; или, с. Сопроводить его полученной вами информацией о предложении распространять соответствующий исходный код. (Эта альтернатива разрешена только для некоммерческого распространения и только в том случае, если вы получили программу в объектном коде или исполняемом виде с таким предложением, в соответствии с вышеизложенным Подразделом b).

Исходный код разработки означает её предпочтительную форму для внесения в нее изменений. Для выполняемой разработки полный исходный код означает весь исходный код для всех модулей, которые он содержит, плюс любые связанные файлы определения интерфейса, плюс скрипты, используемые для управления компиляцией и установкой исполняемого файла. Однако в качестве особого исключения распространяемый исходный код не должен включать в себя ничего, что обычно распространяется (в исходном или двоичном виде) с основными компонентами (компилятором, ядром и т.д.) операционной системы, на которой выполняется исполняемый файл, если только этот компонент сам не сопровождает исполняемый файл.

Если распространение исполняемого или объектного кода осуществляется путем предоставления доступа к копированию из определенного места, то предложение эквивалентного доступа к копированию исходного кода из того же места считается распространением исходного кода, даже если третьи лица не обязаны копировать исходный код вместе с объектным кодом.

4. Вы не имеете права копировать, изменять, сублицензировать или распространять Программу, за исключением случаев, прямо предусмотренных настоящей лицензией. Любая попытка иным образом скопировать, изменить, сублицензировать или распространить Программу является недействительной и автоматически прекращает действие ваших прав в соответствии с настоящей лицензией. Однако для третьих лиц, уже получивших от вас копии или права в соответствии с настоящей лицензией, действие лицензий не будет прекращено до тех пор, пока они остаются в полном соответствии с требованиями.

5. Вы не обязаны принимать эту лицензию, пока вы её не подписали. Однако ничто другое не дает вам разрешения на изменение или распространение Программы или её производных разработок. Эти действия запрещены законом, если вы не принимаете эту лицензию. Таким образом, изменяя или распространяя Программу (или любую разработку, основанную на Программе), вы подтверждаете свое согласие с настоящей лицензией и всеми её условиями копирования, распространения или изменения Программы, или разработок, основанных на ней. 
6. Каждый раз, когда вы распространяете Программу (или любую разработку, основанную на программе), потребитель автоматически получает лицензию от первоначального лица, выдающего разрешение на копирование, распространение или изменение Программы в соответствии с настоящими условиями и положениями. Вы не имеете права налагать какие-либо дополнительные ограничения на осуществление потребителями прав, предоставленных настоящим соглашением. Вы не несете ответственности за обеспечение соблюдения этой лицензии третьими лицами.

7. Если в результате судебного решения или утверждения о нарушении патентных прав или по любой другой причине (не ограничиваясь патентными вопросами) на вас будут установлены требования (будь то по решению суда, соглашению или иным образом), которые противоречат условиям настоящей лицензии, они не освобождают вас от условий настоящей лицензии. Если вы не можете распространять Программу таким образом, чтобы одновременно выполнять свои обязательства по настоящей лицензии и любые другие соответствующие обязательства, то, как следствие, вы не можете вообще распространять Программу. Например, если патентная лицензия не допускает безвозмездного распространения Программы всеми теми, кто получает копии прямо или косвенно через вас, то единственный способ удовлетворить и её, и эту лицензию - это полностью отказаться от распространения Программы.

Если какая-либо часть настоящего раздела будет признана недействительной или не имеющей законной силы при каких-либо конкретных обстоятельствах, то остаток раздела будет оставаться в силе, а раздел в целом будет использоваться при других обстоятельствах.

Цель настоящего раздела не состоит в том, чтобы побудить вас нарушить какие-либо патенты или другие основания на право собственности или оспаривать действительность любых таких претензий; этот раздел имеет единственную цель - защитить целостность системы распространения свободного программного обеспечения, которая реализуется практикой публичного лицензирования. Многие люди внесли существенный вклад В широкий спектр программного обеспечения, распространяемого через эту систему, полагаясь на последовательное применение этой системы; автор / донор должен решить, желает ли он или она распространять программное обеспечение через любую другую систему, и лицензиат не может навязать этот выбор.

Этот раздел предназначен для того, чтобы полностью прояснить, что, как полагают, является следствием остальной части этой лицензии.
8. Если распространение и / или использование Программы ограничено в определенных странах либо патентами, либо защищенными авторским правом интерфейсами, первоначальный правообладатель, помещающий Программу под эту лицензию, может добавить явное ограничение географического распространения, исключающее эти страны (то есть распространение разрешено только в странах или между странами, не исключенными таким образом). В этом случае данная лицензия включает в себя ограничение, как если бы оно было записано в «теле» этой лицензии.

9. Фонд свободного программного обеспечения может время от времени публиковать пересмотренные и/ или новые версии Общедоступной лицензии. Такие новые версии будут похожи по духу на настоящую версию, но могут отличаться в деталях в контексте решения новых проблем или вызовов.

Каждой версии присваивается отличительный номер. Если Программа указывает номер версии лицензии, которая применяется к ней, и «любую более позднюю версию», у вас есть возможность следовать положениям и условиям либо этой версии, либо любой более поздней версии, опубликованной Фондом свободного программного обеспечения. Если Программа не указывает номер версии этой лицензии, вы можете выбрать любую версию, когда-либо опубликованную Фондом свободного программного обеспечения.

10. Если вы желаете включить части Программы в другие бесплатные программы, условия распространения которых отличаются, напишите автору, чтобы попросить разрешения. Для программного обеспечения, защищенного авторским правом Фонда свободного программного обеспечения, напишите в Фонд свободного программного обеспечения; иногда мы делаем исключения для этого. Наше решение будет руководствоваться двумя целями: сохранение свободного статуса всех производных нашего свободного программного обеспечения и содействие совместному использованию и повторному использованию программного обеспечения в целом.

\section{ОТСУТСТВИЕ ГАРАНТИИ}

11. ПОСКОЛЬКУ ПРОГРАММА ЛИЦЕНЗИРУЕТСЯ БЕСПЛАТНО, НА НЕЕ НЕ РАСПРОСТРАНЯЕТСЯ НИКАКИХ ГАРАНТИЙ В ТОЙ МЕРЕ, В КАКОЙ ЭТО РАЗРЕШЕНО ПРИМЕНИМЫМ ЗАКОНОДАТЕЛЬСТВОМ. ЗА ИСКЛЮЧЕНИЕМ СЛУЧАЕВ, КОГДА ИНОЕ УКАЗАНО В ПИСЬМЕННОЙ ФОРМЕ, ПРАВООБЛАДАТЕЛИ И/ИЛИ ДРУГИЕ СТОРОНЫ ПРЕДОСТАВЛЯЮТ ПРОГРАММУ «КАК ЕСТЬ» БЕЗ КАКИХ-ЛИБО ГАРАНТИЙ, ЯВНЫХ ИЛИ ПОДРАЗУМЕВАЕМЫХ, ВКЛЮЧАЯ, НО НЕ ОГРАНИЧИВАЯСЬ ИМИ, ПОДРАЗУМЕВАЕМЫЕ ГАРАНТИИ ТОВАРНОЙ ПРИГОДНОСТИ И ПРИГОДНОСТИ ДЛЯ КОНКРЕТНОЙ ЦЕЛИ. ВЕСЬ РИСК, СВЯ- 
ЗАННЫЙ С КАЧЕСТВОМ И ПРОИЗВОДИТЕЛЬНОСТЬЮ ПРОГРАММЫ, ЛЕЖИТ НА ВАС. ЕСЛИ ПРОГРАММА ОКАЖЕТСЯ НЕИСПРАВНОЙ, ВЫ БЕРЕТЕ НА СЕБЯ РАСХОДЫ НА ВСЕ НЕОБХОДИМОЕ ОБСЛУЖИВАНИЕ, РЕМОНТ ИЛИ ИСПРАВЛЕНИЕ.

12. НИ В КОЕМ СЛУЧАЕ, ЕСЛИ ЭТО НЕ ТРЕБУЕТСЯ ПРИМЕНЯЕМЫМ ЗАКОНОДАТЕЛЬСТВОМ ИЛИ НЕ СОГЛАСОВАНО В ПИСЬМЕННОЙ ФОРМЕ, ЛЮБОЙ ПРАВООБЛАДАТЕЛЬ ИЛИ ЛЮБАЯ ДРУГАЯ СТОРОНА, КОТОРАЯ МОЖЕТ ИЗМЕНЯТЬ И / ИЛИ РАСПРОСТРАНЯТЬ ПРОГРАММУ, КАК ЭТО РАЗРЕШЕНО ВЫШЕ, НЕ НЕСЕТ ОТВЕТСТВЕННОСТИ ПЕРЕД ВАМИ ЗА УЩЕРБ, ВКЛЮЧАЯ ЛЮБЫЕ ОБЩИЕ, СПЕЦИАЛЬНЫЕ, СЛУЧАЙНЫЕ ИЛИ КОСВЕННЫЕ УБЫТКИ, ВОЗНИКАЮЩИЕ В РЕЗУЛЬТАТЕ ИСПОЛЬЗОВАНИЯ ИЛИ НЕВОЗМОЖНОСТИ ИСПОЛЬЗОВАНИЯ ПРОГРАММЫ (ВКЛЮЧАЯ, НО НЕ ОГРАНИЧИВАЯСЬ ЭТИМ, ПОТЕРЮ ДАННЫХ ИЛИ НЕТОЧНОСТЬ ДАННЫХ, УБЫТКИ, ПОНЕСЕННЫЕ ВАМИ ИЛИ ТРЕТЬИМИ ЛИЦАМИ, ИЛИ НЕСПОСОБНОСТЬ ПРОГРАММЫ РАБОТАТЬ С ЛЮБЫМИ ДРУГИМИ ПРОГРАММАМИ), ДАЖЕ ЕСЛИ ТАКОЙ ВЛАДЕЛЕЦ ИЛИ ДРУГАЯ СТОРОНА БЫЛИ ПРЕДУПРЕЖДЕНЫ О ВОЗМОЖНОСТИ ТАКОГО УЩЕРБА.

\section{ОКОНЧАНИЕ УСЛОВИЙ И ПОЛОЖЕНИЙ}

Если вы разрабатываете новую программу и хотите, чтобы она была максимально полезной для общества, лучший способ - это сделать её свободной программой, которую каждый может распространять и изменять в соответствии с этими условиями.

Для этого приложите к программе соответствующие уведомления. Безопаснее всего прикрепить их к началу каждого исходного файла, чтобы наиболее эффективно передать исключение гарантии; и каждый файл должен иметь, по крайней мере, строку «авторское право» и указатель на то, где находится полное уведомление.

<одна строка, чтобы дать название программы и краткое представление о том, что она делает.>

$$
\text { Copyright (C) 19yу <имя автора> }
$$

Эта программа является свободным программным обеспечением; вы можете распространять её и/или изменять в соответствии с условиями GNU General Public License, опубликованной Фондом свободного программного обеспечения; либо версия 2 Лицензии, либо (по вашему выбору) любая более поздняя версия.
Эта программа распространяется в надежде, что она будет полезна, но без каких-либо гарантий; даже без подразумеваемой гарантии товарной пригодности или пригодности для определенной цели. Смотрите стандартную Общедоступную лицензию GNU для получения дополнительной информации.

Вы должны были получить копию GNU General Public License вместе с этой программой; если нет, напишите в Фонд свободного программного обеспечения (Inc.,51 Franklin Street, Fifth Floor, Boston, MA 02110-1301, USA).

Также добавьте информацию о том, как связаться с вами по электронной и бумажной почте.

Если программа интерактивна, сделайте так, чтобы она выводила короткое уведомление (как например, это), когда она запускается в интерактивном режиме:

Gnomovision версия 69, Copyright (C) 19уу имя автора

Gnomovision поставляется без каких-либо гарантий; для получения подробной информации введите «show w». Это бесплатное программное обеспечение, и вы можете распространять его при определенных условиях; введите «show с» для получения подробной информации.

Возможные команды «show w» и «show с» должны показывать соответствующие части Общедоступной лицензии. Конечно, команды, которые вы используете, могут называться как-то иначе; они могут даже быть щелчками мыши или командами меню, то есть всем тем, что подходит вашей программе.

Вы также должны попросить своего работодателя (если вы работаете программистом) или свой университет, или институт (если таковые имеются) подписать «отказ от авторских прав» на программу, если это необходимо. Вот пример; измените имена:

Yoyodyne, Inc., настоящим отказывается от всех авторских прав на компилирующую программу «Gnomovision», написанную Джеймсом Хакером.

<подпись Тая Куна>, 1 апреля 1989 года Тай Кун, вице-президент

\section{ЛИТЕРАТУРА}

1. Dannenberg R. B. Nyquist Reference Manual Version 3.15 // Carnegie Mellon University — School of Computer Science/ Pittsburgh, PA 15213, U.S.A. 11.08. 2018 p.276.

2. Dannenberg R. B. Nyquist Reference Manual Version 2.36 // Carnegie Mellon University — School of Computer Science/ Pittsburgh, PA 15213, U.S.A. 05.03. 2007 p.205. 
3. Dannenberg R.B., «Computer Coordination With Popular Music: A New Research Agenda», in Proceedings of the Eleventh Biennial Arts and Technology Symposium at Connecticut College, March 2008.

4. Dannenberg R.B. A Perspective on Computer Music (Computer Music Journal, Vol. 20, №. 1 (Spring, 1996), pp. 52-56) [электронный ресурс] Published by: MIT Press. (URL: www.jstor.org/stable/3681271).

() Таран Василий Васильевич ( allscience@lenta.ru ).

Журнал «Современная наука: актуальные проблемы теории и практики»

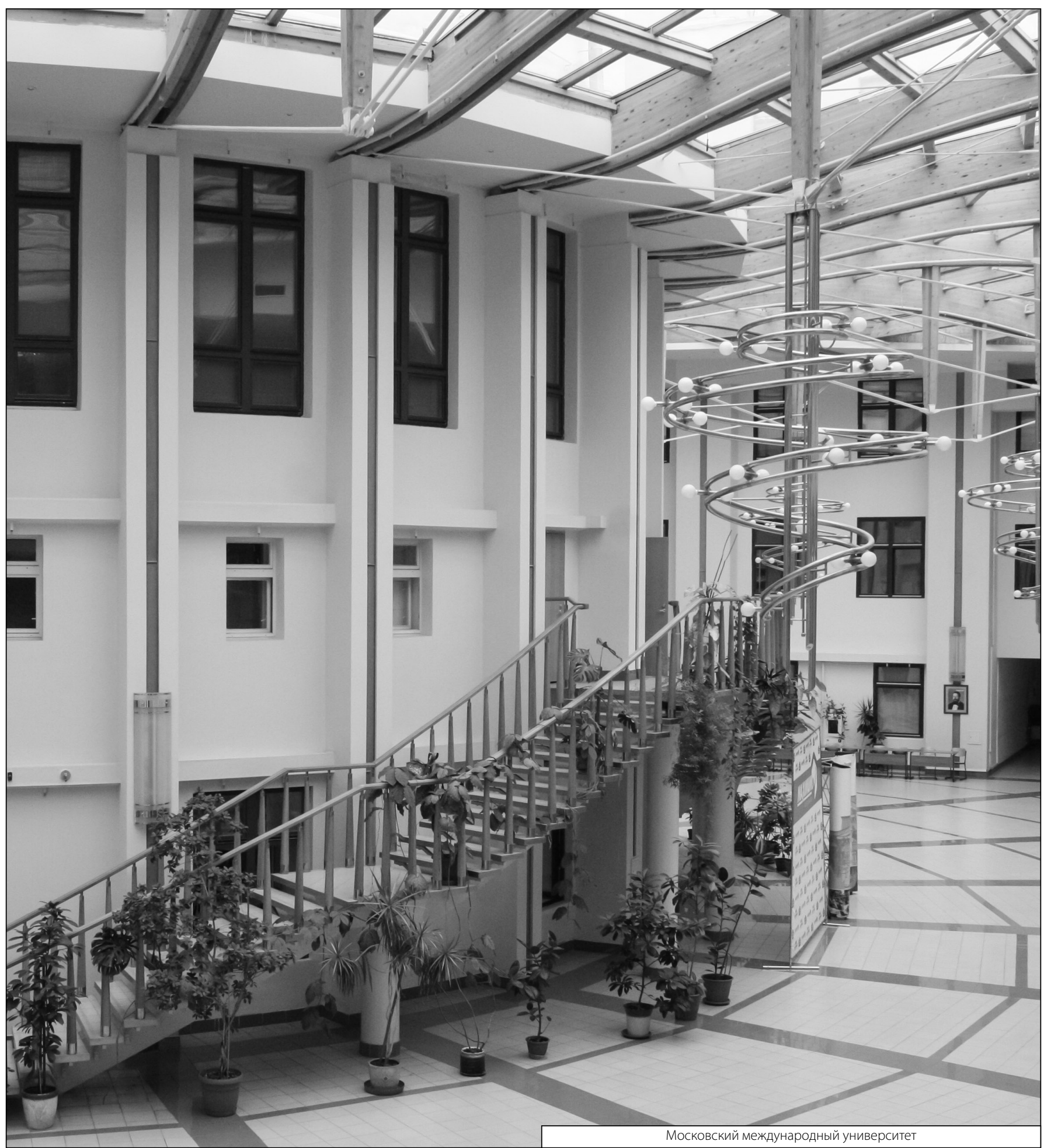

